\title{
Reversed Janus Micro/Nanomotors with Internal Chemical Engine
}

Xing Ma, ${ }^{\ddagger}$ Seungwook Jang, ${ }^{\dagger, \ddagger}$ Mihail N. Popescu, ${ }^{\ddagger}, \perp$ William E. Uspal, ${ }^{\ddagger}, \perp$ Albert Miguel-López, Kersten Hahn, ${ }^{\nabla}$ Dong-Pyo Kim, ${ }^{\dagger, \ddagger}$ and Samuel Sánchez ${ }^{*,+, \|, \S}$

${ }^{\ddagger}$ Max-Planck Institute for Intelligent Systems, Heisenbergstraße 3, 70569 Stuttgart, Germany

${ }^{\dagger}$ Center of Applied Microfluidic Chemistry, Department of Chemical Engineering, Pohang University of Science and Technology, 77 Cheongam-ro, Nam-gu, 37673 Pohang, South Korea

${ }^{\perp}$ IV. Institut für Theoretische Physik, Universität Stuttgart, Pfaffenwaldring 57, D-70569 Stuttgart, Germany

"Institut de Bioenginyeria de Catalunya (IBEC), Baldiri i Reixac 10-12, 08028 Barcelona, Spain

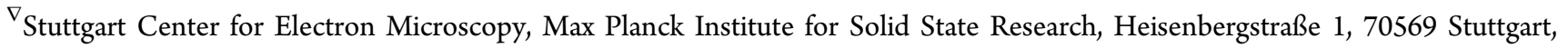
Germany

${ }^{\S}$ Institució Catalana de Recerca i Estudis Avancats (ICREA), Pg. Lluís Companys 23, 08010 Barcelona, Spain

\section{Supporting Information}

ABSTRACT: Self-motile Janus colloids are important for enabling a wide variety of microtechnology applications as well as for improving our understanding of the mechanisms of motion of artificial micro- and nanoswimmers. We present here micro/nanomotors which possess a reversed Janus structure of an internal catalytic "chemical engine". The catalytic material (here platinum $(\mathrm{Pt})$ ) is embedded within the interior of the mesoporous silica $\left(\mathrm{mSiO}_{2}\right)$-based hollow particles and triggers the decomposition of $\mathrm{H}_{2} \mathrm{O}_{2}$ when suspended in an aqueous peroxide $\left(\mathrm{H}_{2} \mathrm{O}_{2}\right)$ solution. The pores/gaps at the noncatalytic $(\mathrm{Pt})$ hemisphere allow the exchange of chemical species in solution between the exterior and the interior of the particle. By varying the diameter of the particles, we observed size-dependent motile behavior in the form of enhanced diffusion for $500 \mathrm{~nm}$ particles, and self-phoretic motion, toward the nonmetallic part, for 1.5 and $3 \mu \mathrm{m}$ ones. The direction of motion was rationalized by a theoretical model based on self-phoresis. For the $3 \mu \mathrm{m}$ particles, a change in the morphology of the porous part is observed, which is accompanied by a change in the mechanism of propulsion via bubble nucleation and ejection as well as a change in the direction of motion.

KEYWORDS: micro/nanomotors, Janus particles, phoretic motion, self-propulsion, mesoporous silica, micromachines
$\mathrm{N}$ amed after the ancient Roman God Janus, micro- or nanosized particles with an architecture of different physical and/or chemical properties over regions of the surface have been widely explored in various areas of science and technology. Typical Janus "motors" have two distinct faces, at least one of which is "active", i.e., catalyzes chemical reactions in the surrounding solution, which leads to self-propulsion. Such motile particles are envisioned to enable multiple, break-through applications. For instance, autonomous Janus motors have been used as an active cargo delivery system to transport microparticles ${ }^{1,2}$ and small molecules ${ }^{3-6}$ as well as active micro/nano platforms for environmental applications. ${ }^{7}$

While there are many methods to fabricate Janus motors, 8 the most common one is deposition, by sputtering or electronbeam (e-beam) evaporation, of a chemically active material, e.g., $\mathrm{Pt}$, onto a monolayer of spherical particles made of silica, polystyerene, etc. ${ }^{10}$ The reverse process of covering the surface of a chemically active particle with an inert material ${ }^{11-13}$ or surface functionalization of chemically active moieties onto one face of an inert Janus particle ${ }^{14,15}$ has also been employed as methods of fabrication for Janus motors. In addition to the well-known catalytic combination of $\mathrm{Pt} / \mathrm{H}_{2} \mathrm{O}_{2}$, many other inorganic chemical reactions, such as $\mathrm{MnO}_{2} / \mathrm{H}_{2} \mathrm{O}_{2},{ }^{16} \mathrm{Al} / \mathrm{H}_{2}{ }^{17}$ $\mathrm{Mg} / \mathrm{H}_{2} \mathrm{O},{ }^{12}$ and $\mathrm{Ir} / \mathrm{N}_{2} \mathrm{H}_{4},{ }^{18,19}$ have been utilized in the design of Janus motors. Biocatalytic reactions triggered by enzymes have been explored recently as a means of achieving propulsion of Janus motors. $3,6,20$

Various mechanisms to convert chemical energy into motility of active colloids have been reported. Bubble propulsion, which can be easily identified by visible gas bubbles detaching from the active side of the motors, ${ }^{1,21-23}$ is one important mechanism for larger motors, i.e., with characteristic size of

Received: July 1, 2016

Accepted: September 6, 2016

Published: September 6, 2016 
Scheme 1. Schematic Illustration of the Steps in the Fabrication of the Reversed Janus Motors and of the Various Motility Scenarios
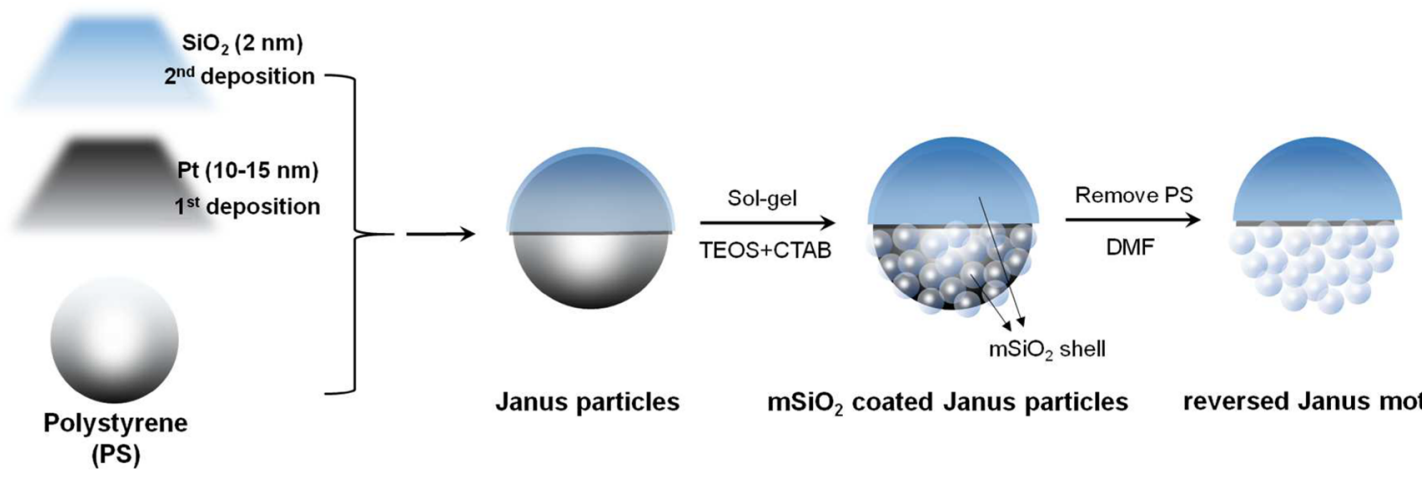

Janus particles $\quad \mathrm{mSiO}_{2}$ coated Janus particles reversed Janus motor

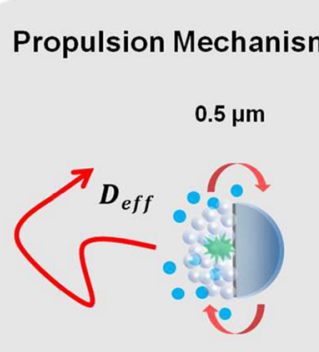

Enhanced Diffusion
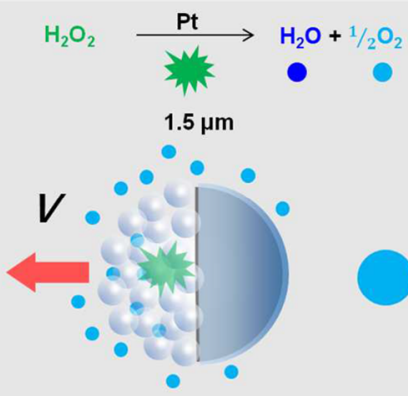

Phoretic Motion
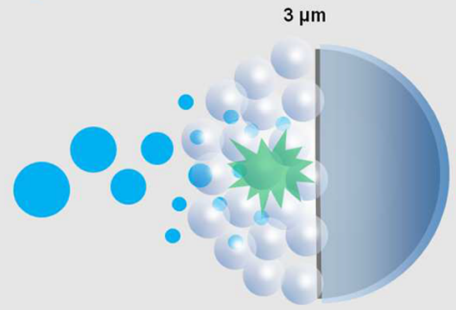

Bubble Propulsion
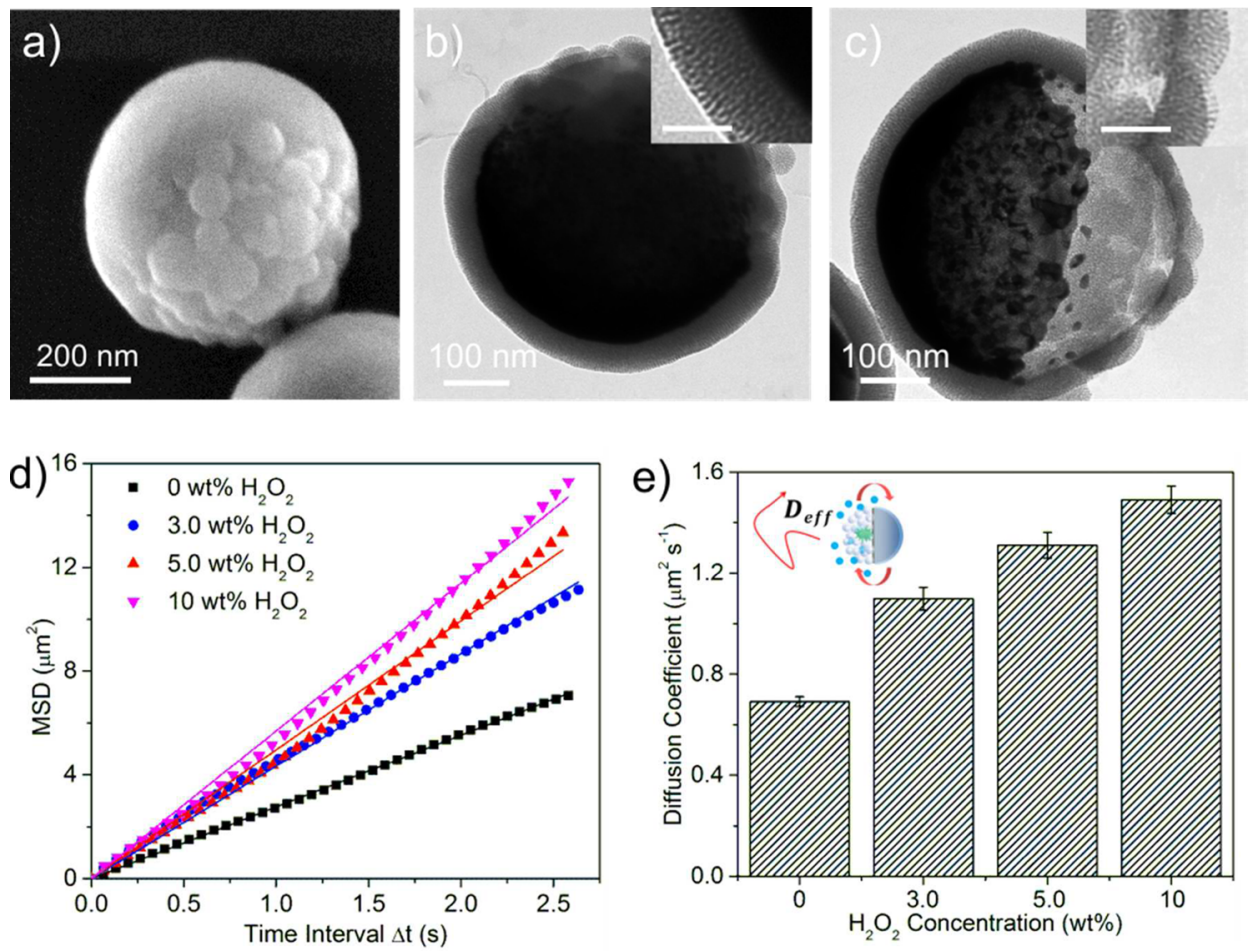

Figure 1. Characterization of the reversed Janus nanomotor $(500 \mathrm{~nm})$ and the dependence of the enhanced diffusion on the concentration of $\mathrm{H}_{2} \mathrm{O}_{2}$ (fuel). (a) SEM and (b) TEM images of the nanomotor before removal of the PS template; (c) TEM image of the nanomotor after removal of the PS template; scale bars in the insets of $(\mathrm{b}$ and c): $50 \mathrm{~nm}$. (d) Average MSD versus time interval $(\Delta t)$ and (e) the corresponding effective (enhanced) diffusion coefficient of the nanomotors at various concentration of $\mathrm{H}_{2} \mathrm{O}_{2}$. The results in $(\mathrm{d}$ and e) are calculated as an average over 10 independent trajectories at each fuel concentration; the error bars represent the standard error of the mean.

tens of micrometers. As the motors' size decreases to a few micrometers, bubble propulsion is seldom reported; in this size range, most of the Janus motors exhibit some form-diffusio, electro, electrochemical- of self-phoresis. ${ }^{10,24,25}$ Although this 
a)
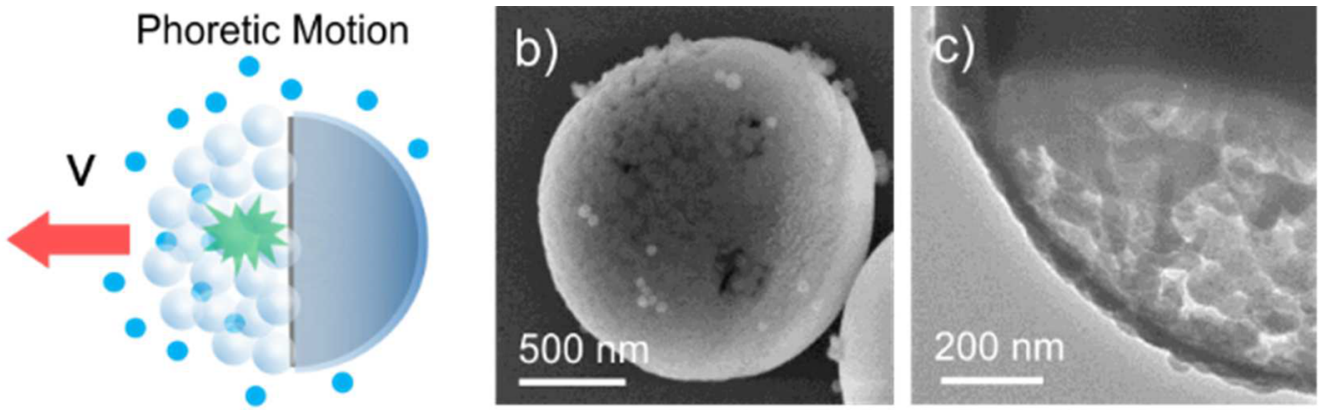

d)
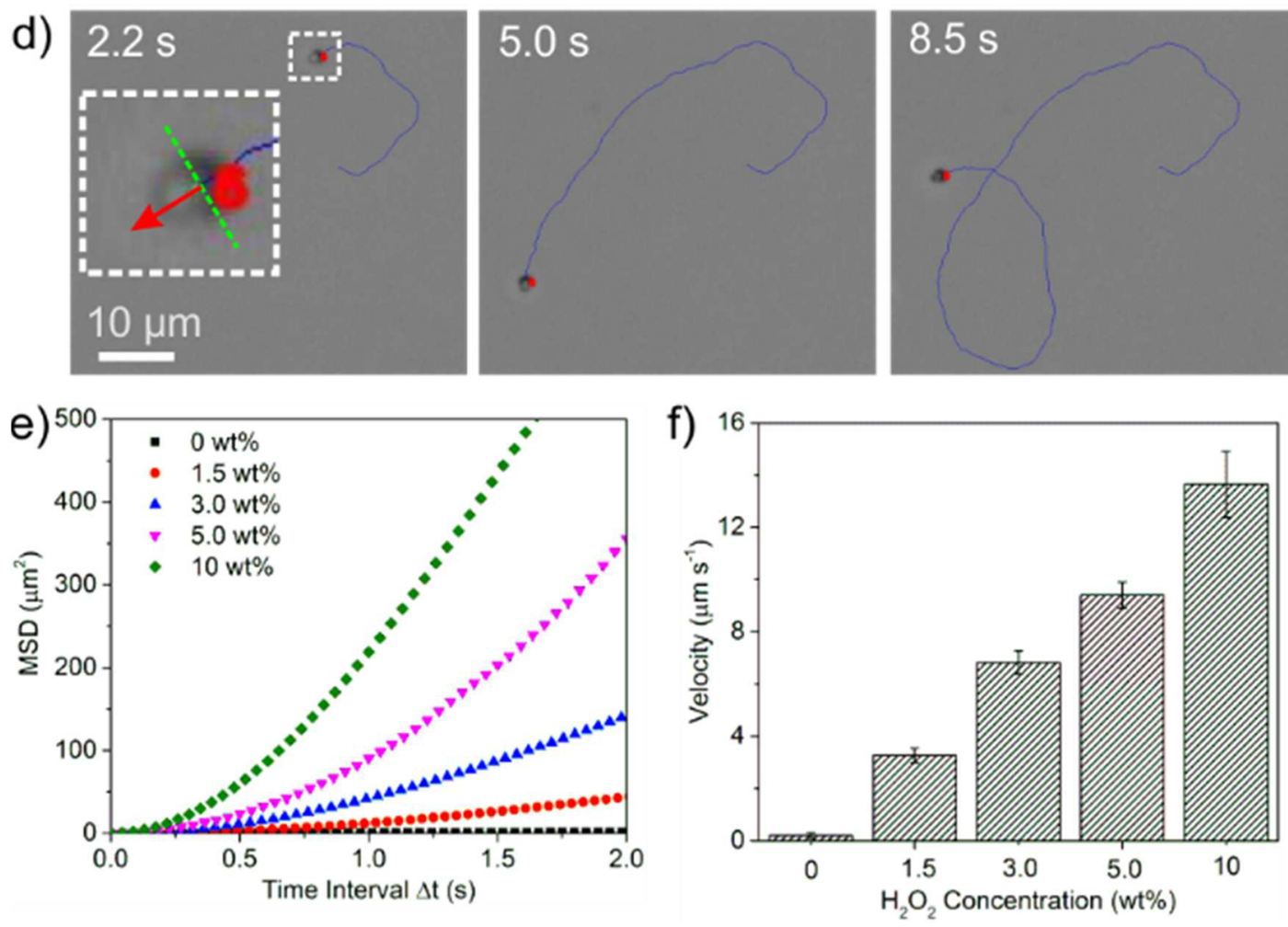

Figure 2. Characterization of the structure and of the motion of the reversed Janus micromotors $(1.5 \mu \mathrm{m})$. (a) Schematic illustration of the motion, assumed to occur by some type of self-phoresis, of the micromotor. (b) SEM of the micromotor and (c) TEM image of the nonmetallic side of the micromotor. (d) Snapshots of a typical trajectory at $10 \mathrm{wt} \% \mathrm{H}_{2} \mathrm{O}_{2}$. Inset: the micromotor moves toward the porous part. The red spot is a label automatically assigned by the tracking software. (e) MSD (average) as a function of the time interval ( $\Delta t)$ and (f) the corresponding average velocity of the micromotor at different fuel concentration. The results in (e and $\mathrm{f}$ ) are calculated as average over 10 independent trajectories at each fuel concentration; error bars show the standard error of the mean.

class of mechanisms was intensively studied in the past decade, recent debates in the literature highlight a number of interesting questions on the self-phoretic motion, which are yet to be answered. $^{24,26-28}$ If the size of the active particle is further decreased toward submicrometer scale, any directionality of the motion is lost due to fast rotational diffusion, and the particles exhibit enhanced diffusion. ${ }^{4,13,29}$

Here, we report on the development of a $\mathrm{mSiO}_{2}$-based hollow, spherical Janus motors with "reversed” Janus structure, in that the catalytic layer $(\mathrm{Pt})$ is placed inside the hollow particles, and thus the catalytic reaction takes place only inside the cavity as well, in contrast to conventional Janus motors (see Scheme 1). By using polystyrene (PS) particles of different sizes as starting templates in the fabrication procedure, we successfully control the size of the motors from $500 \mathrm{~nm}$ up to 3 $\mu \mathrm{m}$. We observed size-dependent motility behavior (see Scheme 1): enhanced diffusion for $500 \mathrm{~nm}$ particles; directional phoretic motion toward the nonmetallic side for 1.5 and $3 \mu \mathrm{m}$ particles, which is rationalized via theoretical modeling; and bubble propulsion toward the metallic side for $3 \mu \mathrm{m}$ particles, for which a change in the morphology of the porous structure of the motor is also noticeable. These Janus motors with catalytic reactions inside the motors' body have the advantage that the whole external surface is available for functionalization, compared to conventional Janus motors where only half of the external surface is available. Furthermore, being made out of $\mathrm{mSiO}_{2}$, the surface can be easily functionalized with various moieties, and therefore they can act as versatile active micro/ nanoplatforms in applications such as environmental remediation and active drug delivery. Via theoretical modeling and analysis, we have shown that the directional motion of the reversed motors, in the absence of bubbles, is compatible with a self-phoretic mechanism. These findings contribute to a better understanding of self-phoresis and to the design and fabrication of other new types of chemically powered micro/nanomotors. 
a)

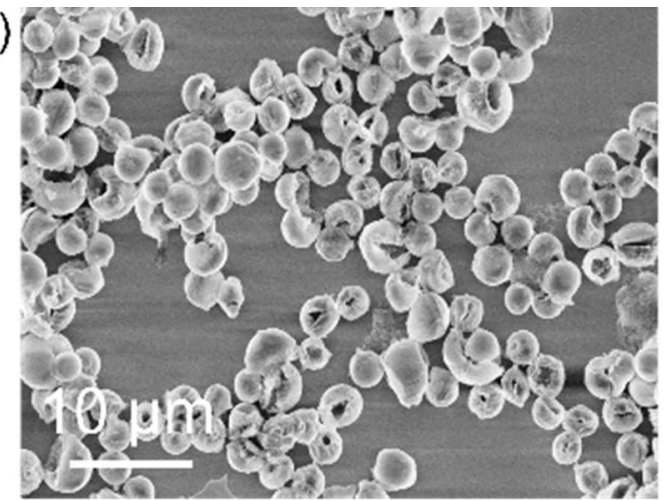

c)

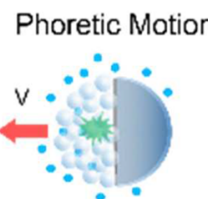

d)

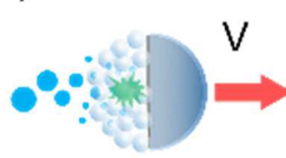

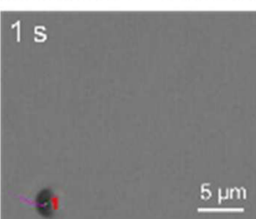

$\underline{5 \mu m}$

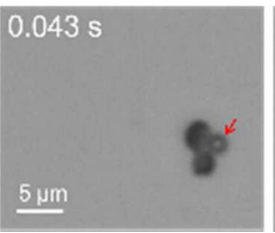

b)
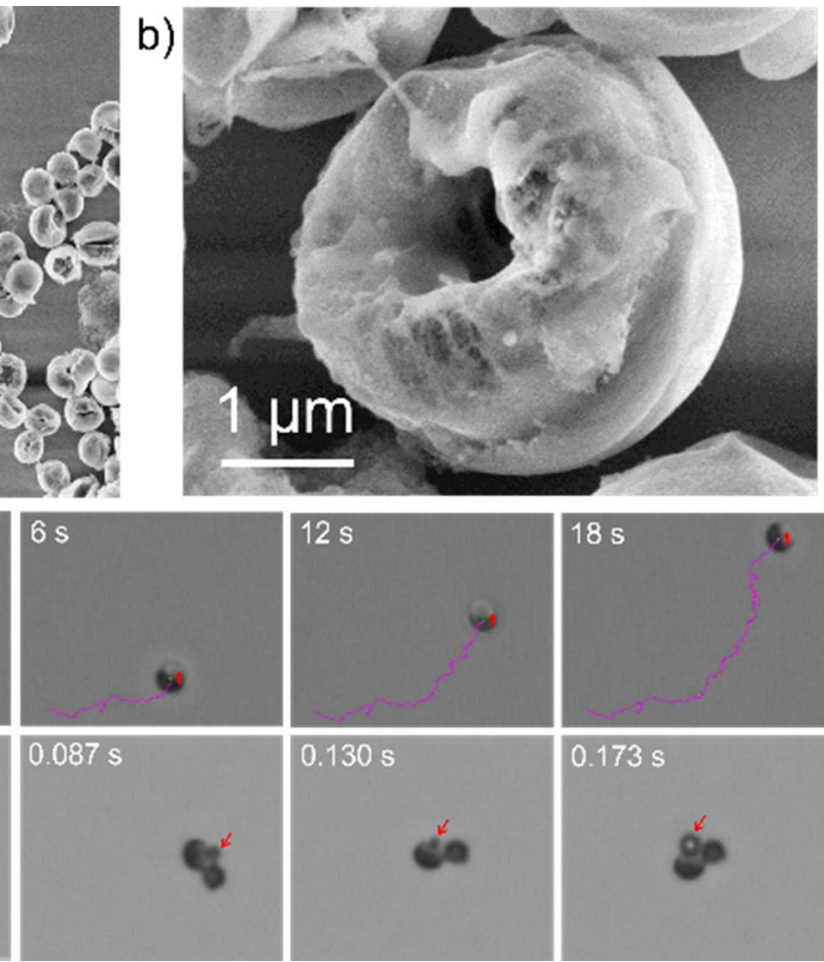

Figure 3. Phoretic motion or bubble propulsion for reversed Janus micromotors of large size ( $3 \mu \mathrm{m})$. (a) Low- and (b) high-magnification SEM images of Janus micromotors $(3 \mu \mathrm{m})$. (c, d) Schematic illustration of the motion mechanism, and snapshots along a typical trajectory, of micromotor $(3 \mu \mathrm{m})$ undergoing (c) directional phoretic motion at 10 wt $\% \mathrm{H}_{2} \mathrm{O}_{2}$ (motion toward the nonmetallic side) and (d) bubble propulsion at 10 wt $\% \mathrm{H}_{2} \mathrm{O}_{2}$ (motion away from the nonmetallic side).

\section{RESULTS AND DISCUSSION}

We start by succinctly describing the fabrication method. Layers of $\mathrm{Pt}(10-15 \mathrm{~nm})$ and $\mathrm{SiO}_{2}(2 \mathrm{~nm})$ are sequentially deposited on a monolayer of (template) PS, spherical particles, yielding typical Janus particles, as depicted in Scheme 1. (SEM images of Janus PS particles of three different sizes after Pt deposition (JPS-Pt) are presented in in Figure S1.) Subsequently, the whole surface is encapsulated, using solgel chemistry, by a mesoporous silica $\left(\mathrm{mSiO}_{2}\right)$ shell; a typical outcome is shown in Figure 1a,b. Due to the different surface properties, hydrophilic $\mathrm{SiO}_{2}$ and hydrophobic PS, the $\mathrm{mSiO}_{2}$ shows a different growth morphology on the two sides. The $\mathrm{mSiO}_{2}$ shell coated on the Pt hemisphere (metallic side) has a uniform and smooth surface with a thickness of about $50 \mathrm{~nm}$ (inset of Figure $1 \mathrm{~b}$ ). However, the $\mathrm{mSiO}_{2}$ coated on the PS hemisphere (nonmetallic side) has a rough surface consisting of aggregated small $\mathrm{mSiO}_{2}$ particles. In the final step of the fabrication, the PS template is removed by dissoving it in dimethylformamide (DMF). In this way we successfully obtain $\mathrm{mSiO}_{2}$-based reversed Janus motors, in which the Pt hemisphere (black color) is embedded inside the hollow $\mathrm{mSiO}_{2}$ shell (Figure 1c) (Pt is black, on the left). We noticed relatively large pores/gaps, with a diameter of up to $50 \mathrm{~nm}$, at the nonmetallic side (inset of Figure 1c), which would allow relatively unhindered diffusion of molecular species, such as $\mathrm{H}_{2} \mathrm{O}_{2}$ and $\mathrm{O}_{2}$, between the solution inside and outside the shell. In contrast to conventional Janus motors, where the $\mathrm{Pt}$ is on the outside of the surface, here the $\mathrm{H}_{2} \mathrm{O}_{2}$ decomposition takes place inside the hollow cavity, acting as internal "chemical engine" for the motile Janus particles.

The self-propulsion of the nanosized motors $(500 \mathrm{~nm}$ diameter) was characterized by optical microscopy observation (see Experimental Section in the SI). Automatic tracking and calculation of mean-square-displacement (MSD) at different time intervals $(\Delta t)$ was accomplished by a self-developed code based on Python (Figure S2). For the small particles, the rotational diffusion coefficient of the symmetry axis of the particle $D_{\mathrm{r}}=\tau_{\mathrm{r}}^{-1}=k_{\mathrm{B}} T /\left(8 \pi \mu R^{3}\right)$, where $k_{\mathrm{B}}$ is the Boltzmann constant, $T$ is the absolute temperature, $\mu$ is the viscosity of the solution, and $R$ is the radius of the nanomotors, is large (note the dependence on $\left.1 / R^{3}\right)$; therefore, the nanomotors have a small characteristic reorientation time $\tau_{\mathrm{r}}=0.092 \mathrm{~s}$ at $22{ }^{\circ} \mathrm{C}$, the ambient temperature at which the experiments have been carried out. Typical examples of the motion of the nanomotors without (Video S1) and with (Videos S2 and S3) fuel, respectively, are provided in the Supporting Information (SI). Due to the rapid reorientation of the symmetry axis of the motile colloid, the active nanomotors are expected to exhibit enhanced diffusion, but very weak, if any, directional motion. ${ }^{4,13}$ This is indeed what our experiments show, in that the MSD depends linearly on time, and its slope increases with increasing concentration of fuel. The effective diffusion coefficient $D_{\text {eff }}$ is extracted from the slope of the linear dependence as MSD $=4 D_{\text {eff }} \Delta t$ (assuming the motion to be two-dimensional, corresponding to trajectories in the focal plane under optical observation). ${ }^{10,30}$ The diffusion coefficient in the absence of fuel is $0.69 \pm 0.02 \mu \mathrm{m}^{2} \mathrm{~s}^{-1}$; this value is slightly lower than the theoretical expectation of $0.86 \mu \mathrm{m}^{2} \mathrm{~s}^{-1}$ following the Stokes-Einstein equation $D=k_{\mathrm{B}} T /(6 \pi \mu R)$. The dependence of $D_{\text {eff }}$ on fuel concentration, summarized in Figure $1 \mathrm{~d}$, shows an increase from the Brownian diffusion value (0.69 $\left.\pm 0.02 \mu \mathrm{m}^{2} \mathrm{~s}^{-1}\right)$ in the absence of fuel to $1.10 \pm 0.04 \mu \mathrm{m}^{2} \mathrm{~s}^{-1}$ (at 3 wt $\% \mathrm{H}_{2} \mathrm{O}_{2}$ ), $1.31 \pm 0.05 \mu \mathrm{m}^{2} \mathrm{~s}^{-1}$ (at 5 wt $\% \mathrm{H}_{2} \mathrm{O}_{2}$ ), and $1.49 \pm 0.05 \mu \mathrm{m}^{2} \mathrm{~s}^{-1}$ (at 10 wt $\% \mathrm{H}_{2} \mathrm{O}_{2}$ ), respectively. 

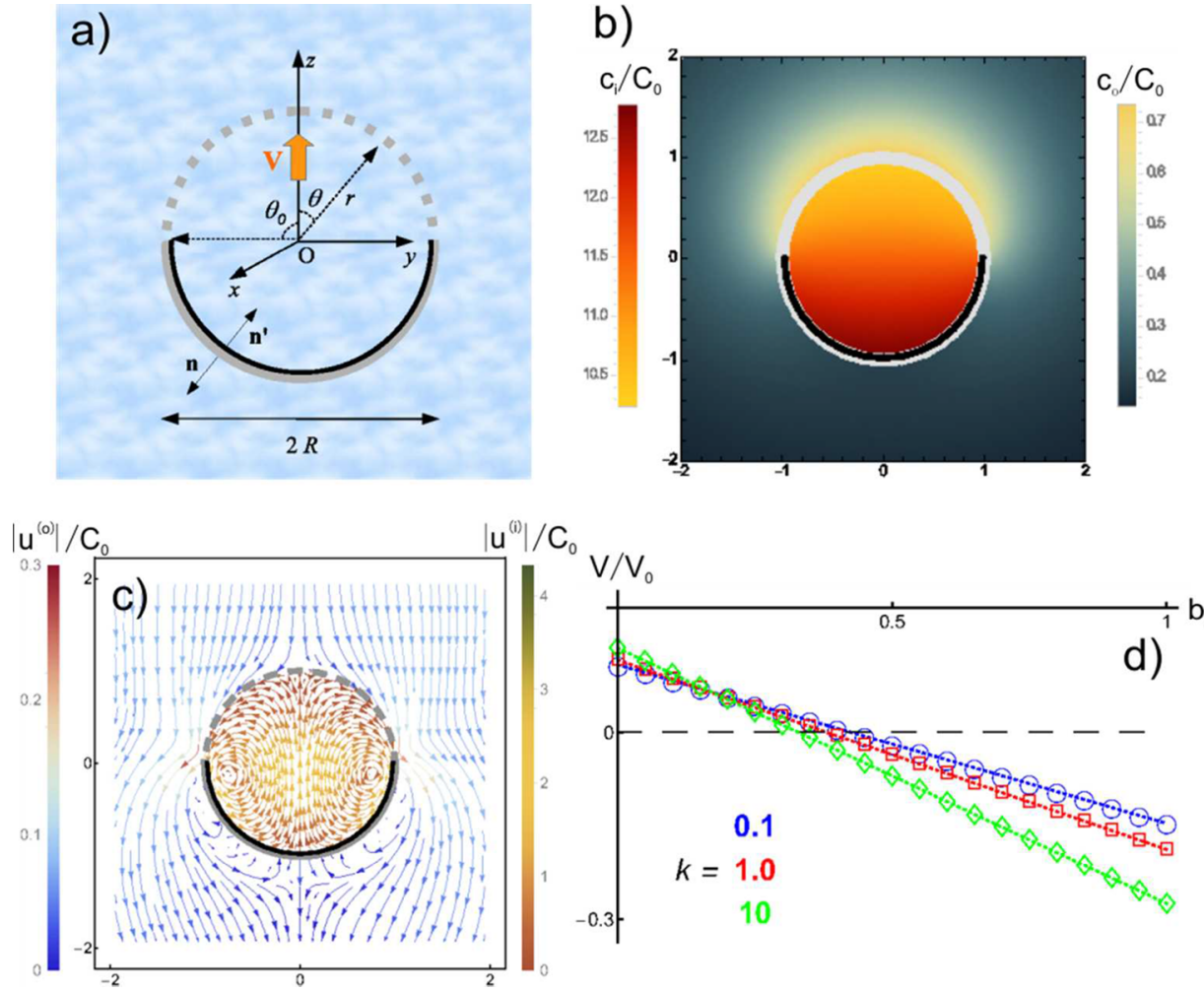

Figure 4. (a) Schematic illustration of the model and notations. (b) Density (scaled by $C_{0}$ ) of solute molecules (color coded) inside (left legend) and outside (right legend) the particle. (c) Flow of the solution (streamlines) in the fixed (laboratory) system when the interior and exterior flows are hydrodynamically coupled by transmission of tangential stress; the color codes the magnitude of the flow velocity (scaled by $V_{0}$ ) inside (right legend) and outside (left legend) the particle, respectively. (d) The velocity of the particle (scaled by $V_{0}$ ) as a function of the ratio $b_{r}$ of phoretic mobilities (see the main text) for various values of the porosity para meter $k$ and slip-lengths (specifying the strength of the interior/exterior hydrodynamic coupling), as given in the main text. (Also see the main text for the definitions of $C_{0}$ and $V_{0}$.)

We now turn to the case of the larger particles of about 1.5 $\mu \mathrm{m}$ diameter. In spite of large pores and gaps at the nonmetallic side (Figure 2b,c), the micromotors maintain an overall spherical shape. Due to their size, these micromotors have a rotational diffusion time of the symmetry axis $\tau_{\mathrm{r}}=2.48 \mathrm{~s}$, which is significantly larger than the relaxation time of the chemical composition of the solution around the colloid by diffusion. In this case, directional self-propulsion of the micromotor (1.5 $\mu \mathrm{m}$ ) is observed (Figure $2 \mathrm{~d}$ ), and the MSD curves now show the corresponding parabolic shapes at short times (Figure 2e). ${ }^{10,29,30}$ The dependence of the velocity on the fuel concentration shown in Figure 2f) further argues for a mechanism of motion involving the catalytic decomposition of $\mathrm{H}_{2} \mathrm{O}_{2}$. The velocity $V$ of the micromotor was calculated directly from the ratio between the length $L$ of the trajectory during a known time period $t$ and the time $t$. Alternatively, the velocity $V$ can be estimated by fitting the parabolic part of the MSD with $4 D \Delta t+V^{2} \Delta t .^{10,30}$ The values thus obtained are similar to the ones directly calculated as above. Snapshots along a typical trajectory are presented in Figure 2d) (see also Video S4). As shown in the inset of the first panel, from these optical observations we can identify the direction of the motion to be toward the nonmetallic side. Since typical, compact core $\mathrm{SiO}_{2} /$ Pt or PS/Pt Janus spherical micromotors (1-2 $\mu \mathrm{m})$ also move toward the noncatalytic part of the Janus structure, this observation raises the possibility that the catalytic reaction actually occurs at the external surface of the Pt hemisphere, via diffusion of the fuel and product species through the very narrow nanochannels of the smooth $\mathrm{mSiO}_{2}$ shell covering the
Pt film (inset of Figure 1b). To test this possibility, we have used motors without removal of the PS core, which thus do not have the "cavity" available for reactions. When such motors are suspended in 5 wt $\% \mathrm{H}_{2} \mathrm{O}_{2}$ solution, we have not observed either indications of $\mathrm{H}_{2} \mathrm{O}_{2}$ catalytic decomposition or motion of the particles (Video S5). Therefore, we rule out this possibility and conclude that the motion is due to chemical reactions occurring inside the cavity of the particle. Further discussion of this case, including a theoretical analysis that provides an explanation for the motility and the direction of motion, is presented below.

By using larger PS particles (about $3 \mu \mathrm{m}$ ) as templates, micromotors with a different morphology of the nonmetallic side are obtained: Instead of a shell with quasi-uniform porosity, now the motor has a shell with one large hole (Figure 3a,b). For this type of micromotor, we observe, at the same fuel concentration ( 10 wt $\% \mathrm{H}_{2} \mathrm{O}_{2}$ ), either phoretic motion or bubble propulsion; snapshots from typical trajectories are shown in Figures 3c,d) (Videos S6 and S7, respectively). The red arrows indicate the location from where the bubbles are released. The coexistence of two mechanisms of motion can be explained by the fact that formation of bubbles depends not only on the $\mathrm{O}_{2}$ concentration reaching the nucleation threshold but also on the surface morphology, the roughness of the pore orifice at the nonmetallic side, and whether the rest of the shell is porous or not (which influences the probability for nucleation due to significantly changing the interior $\mathrm{O}_{2}$ concentration that is "funneled" toward the "hole"). 
On the other hand, the phoretic mechanism remains operational independently of these factors.

In line with previous reports on bubble propulsion, we have observed that under similar concentrations of fuel the velocity of micromotors moving by bubble propulsion is significantly larger (by a factor of 10 in the current experiments) than in the case of phoretic motion. Motion by bubble propulsion can be promoted by adding a surfactant, e.g., sodium dodecyl sulfate (SDS, 0.5 wt \%) to the solution. Upon addition of SDS, we observed very fast velocities of micromotors driven by bubble propulsion (Figure S3 and Video S8), up to about $500 \mu \mathrm{m} \mathrm{s}^{-1}$; at the same time, the phoretic motion was completely suppressed, an effect previously reported ${ }^{31}$ but yet to be conclusively explained. We note that for the smaller micromotors $(1.5 \mu \mathrm{m})$, where the porous structure of the shell is missing the "funneling" large hole, addition of SDS did not lead to bubble propulsion.

We rationalize the directed motion exhibited in the absence of bubble formation by using a simple model of a chemically active colloid and of motion by self-phoresis. The model bears similarities with several studies previously shown to capture qualitatively the phenomenology exhibited by such systems. ${ }^{10,32}$

The particle is modeled as a spherical shell of radius $R$ (see Figure 4a) suspended in a Newtonian liquid solution of viscosity $\mu$. Part of the shell, in the form of a spherical cap of opening angle $\theta_{0} \cong 90^{\circ}$, is porous (dotted gray line), while the rest is compact and smooth (solid gray line). Due to the porous structure, the solution fills the particle interior. We assume that all molecular species in the solution can diffuse (with a reduced diffusion constant) through the pores. On the inside of the compact part there is a thin layer of $(\mathrm{Pt})$ catalyst (solid black line). The catalytic decomposition of peroxide at the $\mathrm{Pt}$ side is modeled as a source of a solute molecule $A$ (in this case $\mathrm{O}_{2}$ ), which releases $A$ molecules with a uniform rate $Q$ per area of catalyst. The solute molecules diffuse in solution with diffusion constant $D$. The steady-state motion of the particle with velocity $\mathbf{V}=V \mathbf{e}_{z}$ (see Figure $4 a$ ) is force- and torque-free (since there are no external fields acting on the particle or on the fluid); furthermore, in the range of velocities that are observed experimentally and in the absence of bubble formation, the flow of the solution is in the low Reynolds number regime, and the diffusion of the molecular solute is dominant over advection, i.e., the Péclet number of the solute is small.

At steady state, and under the assumption that the rotational diffusion of the particle is slow compared to the relaxation of the distribution of solute by molecular diffusion, the density $c(\mathbf{r})$ of solute molecules (in the fixed system of coordinates, shown in Figure 4a), which is chosen such that it is centered at the instantaneous position of the center of the particle) is the solution of the Laplace equation $\nabla^{2} c(\mathbf{r})=0$ subject to appropriate boundary conditions. These are (i) on the catalyst covered region, the current of solute molecules along the normal (into the particle) direction equals the production by the source: $-D \partial_{n} c=Q_{\text {i }}$ (ii) vanishing current along the direction of the normal into the particle over the smooth silica part; (iii) over the porous part, the outgoing (at the cavity side of the porous shell) solute molecules current equals the one outgoing at the outside of the porous shell; the magnitude of this current at the point $\mathbf{r}_{\mathrm{P}}$ on the porous region is assumed to be proportional to the difference in density across the porous shell, i.e., $J\left(\mathbf{r}_{\mathrm{P}}\right)=K\left[c_{\mathrm{i}}\left(\mathbf{r}_{\mathrm{P}}\right)-c_{\mathrm{o}}\left(\mathbf{r}_{\mathrm{P}}\right)\right]$, where $c_{\mathrm{i}, \mathrm{o}}$ denotes the density of solute on the inside and the outside of the particle, respectively. The phenomenological parameter $K$ (units of velocity) is a measure of the porosity of the shell: for small values of $K$, the density of solute on the outside of the shell is much smaller than that on the inside, while for large values of $K$ the two densities are comparable. Physically, $K$ is bounded by the fact that transport of solute molecules through the porous shell cannot be faster than transport through free solution. For a thin shell with thickness $\delta$, an upper bound for $K$ can be estimated as $K_{\max }=D / \delta$. The diffusion problem outlined above can be solved in the standard way via a series representation of $c(\mathbf{r})$ in spherical coordinates (see SI). In Figure $4 \mathrm{~b}$ we show an example of solute density distribution $c(\mathbf{r})$ in units of the characteristic density $C_{0}:=Q R / D$ for $k=0.1$, where the dimensionless porosity parameter $k:=K R / D$.

Due to the effective interaction of the solute molecules with the surface of the colloid, the gradients in solute density along the surface of the particle give rise to stresses in the fluid and thus to hydrodynamic flow. ${ }^{33}$ Over the nonporous surfaces (the Pt covered one and the compact, smooth silica part), these interactions are accounted for by a so-called "phoretic slip" hydrodynamic boundary condition on the surface of the particle; i.e., $\mathbf{u}_{\mathrm{s}}=-b \nabla c$ at the surface, with the phoretic mobility $b<0(b>0)$ for repulsive (attractive) interactions, respectively. ${ }^{33}$ The parameter $b$ can take different values over the surface, $b_{\mathrm{Pt}}$ and $b_{\mathrm{Si}}$; based on previous studies, ${ }^{32}$ we expect both of them to be negative and the ratio $b_{\mathrm{r}}=b_{\mathrm{Si}} / b_{\mathrm{Pt}}<1$.

The role played by the porous part of the surface in the hydrodynamics of the solution distinguishes two scenarios of motion. The first possibility is that it effectively insulates the hydrodynamic flows inside and outside of the shell. In this case, the model maps directly into self-diffusiophoresis of the whole shell plus the solution contained inside. This is driven by the distribution of solute on the outside of the shell, the phoretic mobility $b_{\mathrm{Si}}$ on the compact (smooth) silica part, and the hydrodynamic boundary condition specifying the tangential component of the flow velocity over the porous part. (The assumption of a hydrodynamically insulated interior implies that the radial component of the flow velocity vanishes at all points on the shell.) For simplicity, we assume that over this porous part, an effective phoretic slip boundary condition, characterized by a mobility $b_{\text {por }}$, holds; this parameter is expected to satisfy $b_{\text {por }}<0$ and $\left|b_{\text {por }}\right|<\left|b_{\mathrm{Si}}\right|$, since the porous matrix is silica. Irrespective of the value of $b_{\text {por }}$ within those limits, we obtain $V<0$. This is opposite to the direction of motion observed in the experiments, which conclusively argues against this mechanism as a potential explanation of the experimental observation.

We therefore consider in detail a second possibility: While the porous shell prevents a significant radial flow between the inside and the outside, it still allows for transmission of tangential stress from the inside to the outside of the porous shell. This "spilling-into" scenario has been extensively studied in the context of flow over porous walls, and we model it here as an effective hydrodynamic slip boundary condition ${ }^{34}$ for the hydrodynamic flow $\mathbf{v}(\mathbf{r})$ over the porous part: ${ }^{33} v_{\mathrm{t}}^{(\mathrm{i}, \mathrm{o})}=-\left(\lambda^{(\mathrm{i}, \mathrm{o})} /\right.$ $\mu) \boldsymbol{\sigma}_{r \theta}{ }^{(\mathrm{i})}$. Here $\boldsymbol{\sigma}_{r \theta}$ denotes the $r \theta$ component of the stress tensor $\boldsymbol{\sigma}$ of the Newtonian liquid and the minus sign is due to the fact that the normal to the inner surface of the shell is opposite to the unit vector of the radial direction (see SI). Here the sliplength parameters $\lambda^{(i, o)}$ have rather the meaning of a "leakage depth" of the flow from inside to outside. In the following, we set them to the values $100 \mathrm{~nm}$ (the inside) and $50 \mathrm{~nm}$ (outside), since for the mechanism to be operational, the inside value is expected to be larger than the thickness $(\delta \sim 50 \mathrm{~nm})$ of 
the porous shell, while the outside is expected to be smaller than the inside by about $\delta$. We note that these values are not special, in that we have obtained the same phenomenology described below for a range of values $\lambda^{(i)} \geq 90 \mathrm{~nm}$. The (i) phoretic slip and the hydrodynamic slip boundary conditions, together with (ii) the requirement of vanishing radial flow on the surface of the shell, (iii) the rigid translation of the particle with velocity $\mathbf{V}$, (iv) vanishing flow far away from the particle, (v) incompressibility of the flow, and (vi) force free motion of the particle, completely specify the solution of the Stokes equations (i.e., the flow) and the velocity $V$ of the particle; the details of the calculation are provided in the SI. In Figure 4c we show an example of hydrodynamic flow inside and outside a porous particle which moves with a positive (i.e., toward the nonmetallic side) velocity $V / V_{0} \cong 0.06$ (where $V_{0}:=\left|b_{\mathrm{Pt}}\right| C_{0} /$ $(2 R))$, corresponding to the slip-length values noted above and to $b_{\mathrm{r}}=0.1$.

Once the slip-length parameters are fixed, the velocity depends only on the values of $k$ and $0 \leq b_{\mathrm{r}} \leq 1$. As shown in Figure $4 \mathrm{~d}$, while the dependence on $k$ is rather weak (note, though: under the assumption that the solute remains dissolved and no bubbles are formed), the magnitude and, most importantly, the sign of the velocity depend on the value of $b_{\mathrm{r}}$. Previous studies ${ }^{32}$ indicate that small $(<0.3)$ values of this ratio are required in order to capture the emergence of wallbound steady states of motion for compact (i.e., not porous) silica-Pt particles. In that same range of $b_{\mathrm{r}}$ values, our model predicts motion of the porous particle toward the nonmetallic side, which is in agreement with the experimental observations. This feature provides a welcomed self-consistency check of the model.

\section{CONCLUSIONS}

In summary, we have fabricated, studied experimentally, and theoretically modeled a spherical micro/nanomotor with reversed Janus structure. This consists of a mesoporous silica spherical external shell and a catalytic Pt hemisphere embedded inside the hollow cavity. The porous structure at the nonmetallic side of the motors provides the means for chemical species exchange with the surrounding solution such that the catalytic decomposition of peroxide inside the particle can power self-propulsion. Motors of different diameters $(500 \mathrm{~nm}$, $1.5 \mu \mathrm{m}$, and $3 \mu \mathrm{m}$ ) exhibit distinct types of motion: enhanced diffusion, phoretic motion, and either bubble propulsion or phoretic motion, respectively. The motion by self-phoresis, as well as its direction, has been rationalized by developing a theoretical model which accounts for the chemical reactions taking place inside the cavity and coupling of the hydrodynamic flows inside and outside the cavity.

In addition to the flexibility in the type of motion, these micro/nanomotors have the whole external surface made of mesoporous silica, and thus they can be readily modified with versatile functional moieties. Such features promote the Janus motors with reversed structure in the position of promising candidates for active micro/nanoplatforms capable of fulfilling on-demand tasks, such as environmental remediation or active drug delivery, in liquid environments.

\section{METHODS}

Materials. Tetraethylorthosilicate (TEOS, 99\%), triethanolamine (TEOA, 99\%), cetyltrimethylammonoium bromide (CTAB, 99\%), ethanol (>99\%), 2-propanol, polyvinylpyrrolidone (K-30, > 99.5\%), 2,2'-azon-bis(isobutyronitrile) (AIBN, 98\%), 2,2'-azobis(2-methylpro- pionamidine) dihydrochloride (V-50, 97\%), styrene, and dimethylformamide (DMF, 99\%) were purchased commercially.

Instruments. Scanning electron microscopy (SEM) and transmission electron microscopy (TEM) images were captured by a Zeiss ULTRATM 55 SEM machine at $5 \mathrm{kV}$ and a Zeiss EM 912 TEM machine, respectively. An electron beam evaporation (e-beam) system was used to deposit $\mathrm{Pt}$ and $\mathrm{SiO}_{2}$ layer. Optical videos were taken by inverted Leica optical microscopy with $63 \times$ air objective.

PS $(450 \mathrm{~nm})$ Synthesis. First, polyvinylpyrrolidone (K-30, > 99.5\%) was dissolved in $395 \mathrm{~mL}$ water under vigorous stirring. Then, $40 \mathrm{~g}$ styrene was added into the K-30 solution under stirring. The mixture solution was stirred for 30 min under argon atmosphere before slowing heating up to $70{ }^{\circ} \mathrm{C}$. After the temperature was

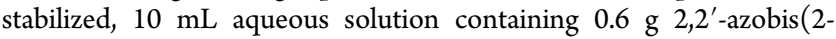
methylpropionamidine) dihydrochloride (V-50) was added in to the mixture solution to initiate the polymerization process. The formed PS particles were collected by centrifugation and washed with deionized (DI) $\mathrm{H}_{2} \mathrm{O}$ and ethanol. The particles were suspended in ethanol for further use.

PS (1.5 and $3 \mu \mathrm{m})$ Synthesis. Typically, we dissolved K-30 ( $1 \mathrm{~g})$ into a mixture solution of iso-propanol $(89 \mathrm{~mL})$ and $\mathrm{DI} \mathrm{H}_{2} \mathrm{O}(10 \mathrm{~mL})$ under vigorous stirring. Then, $2,2^{\prime}$-azon-bis(isobutyronitrile) $(0.34 \mathrm{~g})$ was dissolved in styrene $(10.4 \mathrm{~g})$ and added into the previous solution. After stirring under argon atmosphere for $30 \mathrm{~min}$, the solution was heated up to $70{ }^{\circ} \mathrm{C}$ to initiate the polymerization process. PS $(3 \mu \mathrm{m})$ was prepared by tuning the amount of K-30 $(1.5 \mathrm{~g})$. The formed PS particles were collected by centrifugation and washed with DI $\mathrm{H}_{2} \mathrm{O}$ and ethanol. The particles were suspended in ethanol for further use.

Janus PS Particles Preparation. The ethanol suspension containing PS particles were treated by sonication for $5 \mathrm{~min}$. The particles suspension $(90 \mu \mathrm{L})$ was dropped onto a clean glass slide (50 $\mathrm{mm}$ diameter) that was treated with oxygen plasma for $5 \mathrm{~min}$ beforehand. The glass slide was air-dried, and a monolayer of PS particles would form. A thin layer of $\mathrm{Pt}(10-15 \mathrm{~nm})$ and $\mathrm{SiO}_{2}(2 \mathrm{~nm})$ were sequentially deposited onto the monolayer of PS particles via ebeam evaporation. The Janus particles thus formed were collected by sonication and suspended in DI $\mathrm{H}_{2} \mathrm{O}$.

Reversed Janus Micro/Nanomotors Preparation. The Janus PS particles collected were further suspended in a mixture solution containing DI $\mathrm{H}_{2} \mathrm{O}(2 \mathrm{~mL}), \mathrm{CTAB}(7.5 \mathrm{mg})$, and TEOA $(4 \mathrm{mg})$. The mixture was sonicated for $5 \mathrm{~min}$ and heated up to $80^{\circ} \mathrm{C}$ under stirring. Then, TEOS $(10 \mu \mathrm{L})$ was added into the solution, and the mixture solution was kept at $80{ }^{\circ} \mathrm{C}$ under stirring for $2 \mathrm{~h}$. The mesoporous silica-coated Janus PS particles thus formed were collected by centrifugation and washed with ethanol and $\mathrm{DI} \mathrm{H}_{2} \mathrm{O}$. Then, the PS core was removed by washing the particles with DMF. The PS template was dissolved and removed by repeating the washing process.

Optical Microscopy Observation and MSD Analysis. The movement of the micro/nano motors was observed by inverted optical microscopy (Leica) with $63 \times$ air objective. Aqueous solution containing the micro/nanomotors were placed on a clean glass slides containing varied concentrations of $\mathrm{H}_{2} \mathrm{O}_{2}$. Each video (up to $30 \mathrm{~s}$ long) was recorded with a CCD camera at a frame rate of $20 \mathrm{fps}$. Tracking of the micro/nanomotors were performed by using a home developed code based on Python using the OpenCV library. After obtaining the tracking trajectory of each nanomotors with corresponding coordinates $(x, y)$, the MSD was calculated as $\operatorname{MSD}(\Delta t)=$ $\left\langle\sum_{\{i=x, y\}}\left[r_{\mathrm{i}}(t+\Delta t)-r_{\mathrm{i}}(t)\right]^{2}\right\rangle$ (corresponding to a two-dimensional motion). By varying the time interval $\Delta t$, one obtains the functions $\operatorname{MSD}(\Delta t)$ shown in the various figures in the main text and SI.

\section{ASSOCIATED CONTENT}

\section{Supporting Information}

The Supporting Information is available free of charge via the Internet at The Supporting Information is available free of charge on the ACS Publications website at DOI: 10.1021/ acsnano.6b04358. 
Experimental sections about fabrication of the Janus motors with reversed structure, SEM characterization of Janus particles, video recording, MSD analysis, and details of the theoretical modeling. (PDF)

Videos S1-S4 (ZIP)

Videos S5-S8 (ZIP)

\section{AUTHOR INFORMATION}

\section{Corresponding Author}

*E-mail: sanchez@is.mpg.de, ssanchez@ibecbarcelona.eu.

\section{Author Contributions}

X.M., S.J., D.-P.K., and S.S. initiated this project. X.M. and S.J. prepared the Janus motors with reversed structure and carried out experiments of SEM imaging, optical video recording, and MSD analysis. A.M.-L. developed the code for MSD analysis. M.N.P. and W.E.U. developed the theoretical modeling. K.H. conducted the TEM imaging. S.S. supervised the project. X.M., M.N.P., W.E.U., and S.S. analyzed the results and wrote the paper.

\section{Notes}

The authors declare no competing financial interest.

\section{ACKNOWLEDGMENTS}

The research leading to these results has received funding from the European Research Council under the European Union's Seventh Framework Program (FP7 2007/2013)/ERC grant agreement no. 311529 (LT-NRBS), the European Union Seventh Framework Program [FP7 2007/2013] under grant agreement no. 312483 (ESTEEM2), and the Alexander von Humboldt Foundation (to X.M.). S.S. thanks the Spanish MINECO under grants CTQ2015-68879-R (MICRODIA) and CTQ2015-72471-EXP (Enzwim). D.-P. K. thanks the National Research Foundation (NRF) of Korea grant (NRF-20080061983 and NRF-2014M1A8A1074940).

\section{REFERENCES}

(1) Baraban, L.; Tasinkevych, M.; Popescu, M. N.; Sanchez, S.; Dietrich, S.; Schmidt, O. G. Transport of Cargo by Catalytic Janus Micro-Motors. Soft Matter 2012, 8, 48.

(2) Baraban, L.; Makarov, D.; Streubel, R.; Monch, I.; Grimm, D.; Sanchez, S.; Schmidt, O. G. Catalytic Janus Motors on Microfluidic Chip: Deterministic Motion for Targeted Cargo Delivery. ACS Nano 2012, 6, 3383.

(3) Ma, X.; Sanchez, S. A Bio-Catalytically Driven Janus Mesoporous Silica Cluster Motor with Magnetic Guidance. Chem. Commun. 2015, $51,5467$.

(4) Ma, X.; Hahn, K.; Sanchez, S. Catalytic Mesoporous Janus Nanomotors for Active Cargo Delivery. J. Am. Chem. Soc. 2015, 137, 4976.

(5) Xuan, M.; Shao, J.; Lin, X.; Dai, L.; He, Q. Self-Propelled Janus Mesoporous Silica Nanomotors with Sub-100 nm Diameters for Drug Encapsulation and Delivery. ChemPhysChem 2014, 15, 2255.

(6) Wu, Y.; Lin, X.; Wu, Z.; Möhwald, H.; He, Q. Self-Propelled Polymer Multilayer Janus Capsules for Effective Drug Delivery and Light-Triggered Release. ACS Appl. Mater. Interfaces 2014, 6, 10476.

(7) Jurado-Sánchez, B.; Sattayasamitsathit, S.; Gao, W.; Santos, L.; Fedorak, Y.; Singh, V. V.; Orozco, J.; Galarnyk, M.; Wang, J. SelfPropelled Activated Carbon Janus Micromotors for Efficient Water Purification. Small 2015, 11, 499.

(8) Wang, H.; Pumera, M. Fabrication of Micro/Nanoscale Motors. Chem. Rev. 2015, 115, 8704.

(9) Sánchez, S.; Soler, L.; Katuri, J. Chemically Powered Micro- and Nanomotors. Angew. Chem., Int. Ed. 2015, 54, 1414.
(10) Howse, J. R.; Jones, R. A.; Ryan, A. J.; Gough, T.; Vafabakhsh, R; Golestanian, R. Self-Motile Colloidal Particles: from Directed Propulsion to Random Walk. Phys. Rev. Lett. 2007, 99, 048102.

(11) Gao, W.; Pei, A.; Wang, J. Water-Driven Micromotors. ACS Nano 2012, 6, 8432.

(12) Gao, W.; Feng, X.; Pei, A.; Gu, Y.; Li, J.; Wang, J. Seawaterdriven magnesium based Janus micromotors for environmental remediation. Nanoscale 2013, 5, 4696.

(13) Lee, T.-C.; Alarcón-Correa, M.; Miksch, C.; Hahn, K.; Gibbs, J. G.; Fischer, P. Self-Propelling Nanomotors in the Presence of Strong Brownian Forces. Nano Lett. 2014, 14, 2407.

(14) Zhang, Q.; Dong, R.; Chang, X.; Ren, B.; Tong, Z. SpiropyranDecorated $\mathrm{SiO}_{2}-\mathrm{Pt}$ Janus Micromotor: Preparation and LightInduced Dynamic Self-Assembly and Disassembly. ACS Appl. Mater. Interfaces 2015, 7, 24585.

(15) Pavlick, R. A.; Sengupta, S.; McFadden, T.; Zhang, H.; Sen, A. A Polymerization-Powered Motor. Angew. Chem., Int. Ed. 2011, 50, 9374.

(16) Wang, H.; Zhao, G.; Pumera, M. Beyond Platinum: BubblePropelled Micromotors Based on $\mathrm{Ag}$ and $\mathrm{MnO} 2$ Catalysts. J. Am. Chem. Soc. 2014, 136, 2719.

(17) Gao, W.; D’Agostino, M.; Garcia-Gradilla, V.; Orozco, J.; Wang, J. Multi-Fuel Driven Janus Micromotors. Small 2013, 9, 467.

(18) Dong, R.; Li, J.; Rozen, I.; Ezhilan, B.; Xu, T.; Christianson, C.; Gao, W.; Saintillan, D.; Ren, B.; Wang, J. Vapor-Driven Propulsion of Catalytic Micromotors. Sci. Rep. 2015, 5, 13226.

(19) Gao, W.; Pei, A.; Dong, R.; Wang, J. Catalytic Iridium-Based Janus Micromotors Powered by Ultralow Levels of Chemical Fuels. J. Am. Chem. Soc. 2014, 136, 2276.

(20) Ma, X.; Jannasch, A.; Albrecht, U.-R.; Hahn, K.; Miguel-López, A.; Schäffer, E.; Sánchez, S. Enzyme-Powered Hollow Mesoporous Janus Nanomotors. Nano Lett. 2015, 15, 7043.

(21) Wu, Y.; Wu, Z.; Lin, X.; He, Q.; Li, J. Autonomous Movement of Controllable Assembled Janus Capsule Motors. ACS Nano 2012, 6 , 10910.

(22) Wu, Y.; Si, T.; Lin, X.; He, Q. Near Infrared-Modulated Propulsion of Catalytic Janus Polymer Multilayer Capsule Motors. Chem. Commun. 2015, 51, 511.

(23) Wang, S.; Wu, N. Selecting the Swimming Mechanisms of Colloidal Particles: Bubble Propulsion versus Self-Diffusiophoresis. Langmuir 2014, 30, 3477.

(24) Golestanian, R. Liverpool, T. B.; Ajdari, A. Propulsion of a Molecular Machine by Asymmetric Distribution of Reaction Products. Phys. Rev. Lett. 2005, 94, 220801.

(25) Wang, W.; Duan, W. T.; Ahmed, S.; Mallouk, T. E.; Sen, A. Small Power: Autonomous Nano- and Micromotors Propelled by SelfGenerated Gradients. Nano Today 2013, 8, 531.

(26) Golestanian, R. Anomalous Diffusion of Symmetric and Asymmetric Active Colloids. Phys. Rev. Lett. 2009, 102, 188305.

(27) Jiang, H.-R.; Yoshinaga, N.; Sano, M. Active Motion of a Janus Particle by Self-Thermophoresis in a Defocused Laser Beam. Phys. Rev. Lett. 2010, 105, 268302.

(28) Uspal, W. E.; Popescu, M. N.; Dietrich, S.; Tasinkevych, M. SelfPropulsion of a Catalytically Active Particle Near a Planar Wall: from Feflection to Sliding and Hovering. Soft Matter 2015, 11, 434.

(29) Ke, H.; Ye, S. R.; Carroll, R. L.; Showalter, K. Motion Analysis of Self-Propelled Pt-Silica Particles in Hydrogen Peroxide Solutions. J. Phys. Chem. A 2010, 114, 5462.

(30) Dunderdale, G.; Ebbens, S.; Fairclough, P.; Howse, J. Importance of Particle Tracking and Calculating the Mean-Squared Displacement in Distinguishing Nanopropulsion from Other Processes. Langmuir 2012, 28, 10997.

(31) Simmchen, J.; Magdanz, V.; Sanchez, S.; Chokmaviroj, S.; RuizMolina, D.; Baeza, A.; Schmidt, O. G. Effect of Surfactants on the Performance of Tubular and Spherical Micromotors - a Comparative Study. RSC Adv. 2014, 4, 20334.

(32) Simmchen, J.; Katuri, J.; Uspal, W. E.; Popescu, M. N.; Tasinkevych, M.; Sanchez, S. Topographical Pathways Guide Chemical Microswimmers. Nat. Commun. 2016, 7, 10598. 
(33) Anderson, J. L. Colloid Transport by Interfacial Forces. Annu. Rev. Fluid Mech. 1989, 21, 61.

(34) Happel, J.; Brenner, H. Low Reynolds number hydrodynamics. With special applications to particulate media, 2d ed.; Noordhoff International Publishing: Leiden, 1973; p 569. 